\title{
Agiles Verwaltungsmanagement und interne Kommunikation: Neue Perspektiven einer kommunikationszentrierten Führung in der öffentlichen Verwaltung
}

\section{Susanne Knorre}

Die Kommunikation mit organisationsinternen Stakeholdern, gemeinhin interne Kommunikation genannt, ist in der letzten Dekade vom Rand ins Zentrum der strategisch gesteuerten Kommunikation gerückt. Aus der Praxissicht macht sich der festgestellte Bedeutungszugewinn (Zerfass et al. 2018; Dörfel et al. 2016) an einer Vielzahl von Indikatoren fest, angefangen von steigenden Mitarbeiterzahlen über die zunehmenden Weiterbildungsangebote bis hin zu den vielen inzwischen erhältlichen Praxishandbüchern. Berufsfeldstudien zeigen darüber hinaus, dass im Zuge der Digitalisierung dem Erhalten von Vertrauen größte Bedeutung zugewiesen wird (Zerfass et al. 2018; Edelman 2017). Verbunden wird dies mit der Feststellung, dass Mitarbeitende im Gegensatz zu den Top-Führungskräften als vertrauenswürdig angesehen werden und deshalb als ,Vertrauensbotschafter aktiviert werden müssen, wenn die betreffenden Organisationen insgesamt das Vertrauen ihrer Stakeholder zurückgewinnen wollen.

Aus der theoretischen Perspektive ist dieser Bedeutungszuwachs vor allem auf die Verortung der internen Kommunikation als führungsunterstützende Managementdisziplin (Buchholz und Knorre 2012; Huck-Sandhu 2016) zurückzuführen. Mit der Verbindung zwischen der internen Kommunikation und dem Konzept eines kommunikationszentrierten Managements (Buchholz und Knorre 2019) etabliert sich die interne Kommunikation noch stärker als integraler Bestandteil der

\footnotetext{
S. Knorre $(\bowtie)$

Institut für Kommunikationsmanagement, Hochschule Osnabrück, Campus Lingen, Lingen (Ems), Deutschland

E-Mail: s.knorre@fh-osnabrueck.de
} 
Unternehmensführung. Das gilt umso mehr, als diese nunmehr den Anspruch der Agilität erhebt, d. h. schnell und zugleich sicher auf immer neue, unvorhergesehene Herausforderungen der Systemumwelt reagieren zu können.

Diese Entwicklung der internen Kommunikation wurde bislang vorrangig in Bezug auf privatwirtschaftliche Organisationen beschrieben. Zweifelsohne trifft aber auch die öffentliche Verwaltung zumindest auf sehr vergleichbare Herausforderungen für ihre Leistungsziele, die sich aus allgemein bekannten Merkmalen großer Organisationen wie starren Hierarchien, Silobildung, langen Berichtswege oder Formalismus bei gleichzeitig geringer Mobilisierung der Mitarbeitenden ergeben. Darüber hinaus sind öffentliche Organisationen ebenfalls damit konfrontiert, dass sich ihre von Stakeholdern gestaltete Umwelt stark verändert hat und sich nicht zuletzt aufgrund der weiteren Digitalisierung aller Arbeits- und Lebenswelten weiter verändern wird.

Wenn es also Organisationen der öffentlichen Verwaltung mit Herausforderungen $\mathrm{zu}$ tun haben, die mit denen privatwirtschaftlicher Organisationen vergleichbar sind, dann stellt sich die Frage, wie sich die für Unternehmen entwickelten Handlungskonzepte der internen Kommunikation bzw. der kommunikationszentrierten Führung (Rüegg-Stürm und Grand 2017; Buchholz und Knorre 2019) auf die öffentliche Verwaltung übertragen bzw. dafür adaptieren lassen, um vor allem die Effektivität (und erst nachrangig die Effizienz) von Behörden in ihrer veränderten Umwelt zu stärken. Dieser Fragestellung soll im Folgenden nachgegangen werden.

\section{1 Öffentliche Verwaltung im VUCA-Umfeld: Neue Bedingungen für Verwaltungshandeln}

Die öffentliche Verwaltung insgesamt, besonders aber Behörden, die ihre Aufgaben in wirtschaftsnahen Bereichen wahrnehmen, sind wie alle anderen Organisationen damit konfrontiert, dass ihre Umwelt zunehmend volatil, unsicher, chaotisch und vieldeutig ist. Kennzeichnend für diese sogenannte VUCA-Welt ist vor allem, dass $\mathrm{zu}$ meisternden Herausforderungen oft unerwartet kommen, sich als wenig stabil erweisen und ihre Zeitdimensionen nur schwer eingeschätzt werden können (Volatility). Wann sich eine Veränderung anbahnt und wie sie dimensioniert ist, ist ebenfalls unsicher (Uncertainty). Gleichzeitig sind die Veränderungen von Komplexität (Complexity) geprägt, da ihre Muster nicht wieder wiederholbar, ihre Akteure nicht kontrollierbar und ihre vielschichtigen Dimensionen und Auswirkungen nicht voraussagbar sind (Mack et al. 2016, S. 11, 61 f.). Kausalitäten sind dementsprechend unklar bzw. mehrdeutig oder widersprüchlich (Ambiguity), ohne dass allerdings eine ihnen zugrunde liegende Logik ausgeschlossen werden 
kann. Sie sind offen für vielfältige Interpretationen, die nebeneinander existieren. Die besondere Komplexität dieser Umwelt besteht aber darin, dass in der VUCAWelt alle genannten Merkmale gleichzeitig zu beobachten sind. Zugleich bilden unzählige Einflussgrößen ein vernetztes, kaum überschaubares Ganzes. Jede Einzelentscheidung zieht in einem solchen Umfeld weitere hochkomplexe Wirkungen nach sich, die ihrerseits wiederum zu erneuten Entscheidungen zwingen (Buchholz und Knorre 2019, S. 24 ff.).

Im Gegensatz zu dieser Sichtweise bewertet die in der Tradition von Max Weber stehende rationalitätsfokussierte Verwaltungslehre - ebenso wie die traditionelle Managementlehre - ihre Aufgaben vorrangig aus der Perspektive der Planbarkeit und Rationalität. Sämtliche Pläne bzw. Entscheidungen lassen sich demnach mit dem richtigen Expertenwissen steuern und umsetzen, sodass für Verlässlichkeit und Überschaubarkeit gesorgt ist. Strikt hierarchisch organisierte Organisationen streben die Überwindung komplexer, unübersichtlicher Situationen an. Ihre geordneten, regelbasierten und auf Routinen ausgelegten Strukturen und Kommunikationswege sind auf Beherrschung und Kontrolle ausgerichtet.

Tatsächlich hat sich dieses planzentrierte Handlungs- und Organisationskonzept in der Vergangenheit als durchaus erfolgreich erwiesen. Es ist damit gelungen, „die Komplexität von Beziehungen und Abläufen auf ein handhabbares Maß zu reduzieren, allerdings um den Preis, dass wichtige Aspekte der Wirklichkeit ausgeblendet wurden und die Anpassungsfähigkeit des Systems an Veränderungen und Bedingungen stark eingeschränkt wurden“ (Döring-Seipel und Lantermann 2015, S. 26). Vor dem Hintergrund dieses Befundes stellt sich die Frage, wie die öffentliche Verwaltung organisiert und geführt werden muss, um angemessen auf die neuen, eben skizzierten Umweltbedingungen reagieren zu können.

Unter VUCA-Voraussetzungen scheint es sinnvoll, die traditionelle Handlungsperspektive des plangesteuerten Managements von Organisationen grundsätzlich zu verändern. Anstatt Komplexität bewältigen oder gar reduzieren zu wollen, geht es nun darum, sie quasi zu kultivieren (Buchholz und Knorre 2019, S. 25). Komplexität wird als konstitutiv für erfolgreiches organisationales Handeln betrachtet und weniger als lästiger oder gar belastender Störfaktor. Voraussetzung dafür ist ein in Organisationen geteiltes Bewusstsein dafür, dass nicht mehr alle Situationen und Entwicklungen kontrolliert werden können, sondern es geboten ist, auf Unterstützung und Zusammenarbeit in allen Stakeholderbeziehungen, den internen wie den externen, zu setzen.

Deshalb ist es wenig überraschend, dass neue Qualitätsstandards für öffentliche Verwaltung analog, ja geradezu wortgleich zu den Kriterien entwickelt werden, die auch für Unternehmen in der VUCA-Umwelt gelten (Hill 2014, 2017). Auch öffentliche Verwaltung beantwortet die Frage neu, wie sie ausreichend 
auf unerwartete Probleme reagieren kann, um sich nicht nur als widerstandsfähig (resilient) zu erweisen, sondern sogar noch gestärkt aus unübersichtlichen, risikoreichen Situationen hervorgehen zu können. Bekanntestes Beispiel für diese sektorenübergreifende Neuorientierung ist die aktuelle Fassung des europäischen Qualitätsmanagements EFQM (2013). In diesem Modell wird die Exzellenz von Organisationen inzwischen von Kriterien bestimmt, die vorrangig auf das Ziel einer strategischen und operativen Agilität ,einzahlen“ (Buchholz und Knorre 2019, S. 149 f.; Hill 2017, S. 2 f.).

Dazu wurde in das neue Grundkonzept, mit dem exzellente Unternehmensführung beschrieben wird, das Kriterium „,managing with agility“ (EFQM 2013) aufgenommen. Exzellenz zeichnet sich demnach nicht mehr (nur) dadurch aus, dass es der Organisation gelingt, ihre jeweiligen Geschäfts- und Unterstützungsprozesse wirkungsvoll zu steuern. Nunmehr ist nachzuweisen, dass und wie eine Organisation in der Lage ist, Veränderungen in angemessener Geschwindigkeit zu bewältigen. Die aktuelle Antwort der Managementlehre auf die Herausforderungen der VUCA-Umwelt lautet also Agilität. Agile Organisationen zeichnen sich dadurch aus, dass sie wachsam gegenüber Veränderungen sind und ihre Strukturen so durchlässig und zugleich kollaborativ gestalten, dass Anpassungen an sich verändernde Anforderungen leicht umgesetzt werden können. „Verwaltungen müssen insgesamt eine ,Kultur der Beweglichkeit" entwickeln, die durch Offenheit und Wachheit für neue Entwicklungen gekennzeichnet ist“ (Hill 2017, S. 7).

Hinzu kommt aber noch eine weitere Beobachtung zu den Gründen, warum in der öffentlichen Verwaltung die Suche und Anwendung neuer Qualitätsstandards und Managementmodelle auf der Agenda steht. Denn nach Jahrzehnten der Verwaltungsmodernisierung unter den Überschriften Public Management oder Neue Steuerungsmodelle ist in den letzten Jahren eine Ernüchterung eingekehrt, die nicht zuletzt mit den eher mäßigen Evaluationsergebnissen dieser lange dominierenden Denkschule zu tun hat (Bogumil 2010, S. 114 ff.; Koch 2010, S. 18 f.). Auch wenn diese Debatte sicher noch nicht abgeschlossen ist, lässt sich bereits festhalten, dass der Vorrang des Effizienzprinzips, der mit dem Begriff der Verwaltungsmodernisierung verbunden war, inzwischen einer ausgewogeneren Betrachtung gewichen ist, in der das Effektivitätsprinzip wieder zu alter Relevanz zurückfindet (Bogumil 2010, S. 121 f.).

Dies hängt zweifelsohne damit zusammen, dass die Rollenzuschreibungen für die öffentliche Verwaltung nicht mehr nur auf den Dienstleistungsgedanken (,Public Service') abstellen, sondern dass neue Rollen wie Verhandlungsführer, Moderator, Mediator oder Berater und Impulsgeber für externe Stakeholder, wie für die Politik, an Bedeutung gewonnen haben (Bogumil 2010, S. 122). Weitere Rollen bzw. Aufgaben kommen im Zuge der Digitalisierung hinzu, etwa die eines 
Open Data-Providers, der Daten in geeigneter Qualität quasi als Infrastruktur für private und öffentliche Anwendungen, zum Beispiel zu Forschungszwecken, zur Verfügung stellt (Goldstein und Dyson 2013). Diese Rollenvielfalt ist wiederum eine Folge der eben geschilderten VUCA-Umweltbedingungen, die ganz grundsätzlich infrage stellen, dass Behörden einem möglichst umfassenden Steuerungsanspruch überhaupt gerecht werden können. Effizienter Verwaltungsservice stellt sich deshalb zunehmend als ein Hygienefaktor dar, während die Bewältigung komplexer Aufgaben, die mit der Gestaltung von vertrauensvollen, den gesellschaftlichen Frieden garantierenden Stakeholder-Beziehungen zusammenhängen, die neue Leistung von öffentlicher Verwaltung darstellt.

Das bedeutet aber zugleich, dass die öffentliche Verwaltung mit denselben Paradoxien zu leben bzw. zu kämpfen hat wie Unternehmen auch. Denn bisher verfolgte Verwaltungs- bzw. Steuerungsmodelle werden nicht einfach durch neue abgelöst, sondern alle bestehen gleichzeitig, meist sogar innerhalb einer Organisation. Althergebrachte Verwaltungsprinzipien wie formale Korrektheit, Gleichbehandlung oder Vertrauensschutz sind ebenso zu verfolgen wie neue Handlungsprinzipien, also z. B. Veränderungsfähigkeit, Flexibilität oder kurzfristige Richtungswechsel. Traditionelle Planungs- und Kontrollprozesse werden nicht überflüssig, sondern ergänzt durch inkrementelle und iterative Verbesserungen und das Denken und Handeln in Kontingenzen, d. h. die permanente Auseinandersetzung mit möglichen Alternativen. Der einzige Unterschied dürfte sein, dass diese paradoxen Herausforderungen, d. h. der Umgang mit sich grundsätzlich ausschließenden, widersprüchlichen Handlungserfordernissen, für die öffentliche Verwaltung noch eklatanter ausfallen. Denn in der öffentlichen Verwaltung sind die auf Stabilität und Verlässlichkeit angelegten, mit großem Beharrungsvermögen ausgestatteten Strukturen als noch ausgeprägter einzuschätzen als in privatwirtschaftlichen Organisationen.

Während letzteres vor allem eine erfahrungsbasierte Hypothese darstellt, lässt sich rein logisch aus dem Konzept der Agilität herleiten, dass es in der öffentlichen Verwaltung wie in Unternehmen nicht darum gehen kann, solche Paradoxien wie man es jahrzehntelang im Public-Management-Modell versucht hat - aufzulösen, sondern sie als basales Merkmal von Führung (und Teil der VUCA-Welt) $\mathrm{zu}$ akzeptieren. Ziel ist nicht der allenfalls theoretisch begründbare Anspruch, paradoxe Anforderungen in die eine oder andere Richtung aufzulösen, d. h. das Paradoxon insgesamt zugunsten einer einfachen, eindeutigen Handlungsmaxime zu überwinden. Stattdessen besteht die neue Zielvorstellung darin, die Organisation und ihre Mitarbeitenden durch die paradoxen Bedingungen hindurch zu navigieren, wenn nötig im Zickzackkurs. Das ist einerseits ein befreiender Gedanke, andererseits bedeutet er eben auch zu akzeptieren, dass solche Paradoxien bzw. Unvereinbarkeiten grundsätzlich mit Effizienzverlusten behaftet sind. 


\section{$2 \quad$ Agiles Management: Von der Methode zum Führungskonzept}

Ausgangspunkt für die organisationsübergreifende Entfaltung des Agilitätskonzeptes ist die Betrachtung von Agilität als Handlungskonzept der Digitalisierung. Diese grundsätzliche Aussage lässt sich für alle Organisationen treffen, also auch für die öffentliche Verwaltung. Mit dem E-Government-Gesetz von 2013 sowie dem Onlinezugangsgesetz von 2017 besteht eine anspruchsvolle Vorgabe für die Digitalisierung der öffentlichen Verwaltung in Deutschland. So verpflichtet das Onlinezugangsgesetz Bund und Länder, ihre Verwaltungsportale zu einem Portalverbund zu verknüpfen. Ziel ist es, dass Bürger und Unternehmen über ein einziges Benutzerkonto alle Anliegen bei den Behörden von Bund, Ländern und Kommunen online erledigen können. Fast 600 Verwaltungsleistungen sollen bis 2022 über die gemeinsame Portalplattform erfasst werden. Dies bedeutet eine enorme organisatorische Anstrengung für alle Bereiche der Verwaltung, ausgehend von der IT.

In der IT-Entwicklung der öffentlichen Verwaltung werden deshalb notwendigerweise agile Methoden eingesetzt, nicht zuletzt vorangetrieben vom Bundesverwaltungsamt in verschiedenen Projekten, z. B. dem Wissensmanagementsystem OfficeNet (Bundesverwaltungsamt 2015; Kortendick und Dyrks 2018). Wird von agiler Verwaltung gesprochen (Bartonitz et al. 2018), bezieht sich diese Beschreibung bislang fast ausschließlich auf die Methodenperspektive (BearingPoint 2015; Levesque und Steinbrecher 2017). In den herangezogenen Befragungen werden insbesondere Workflow-Management-, Kollaborations- und Wissensmanagement-Plattformen als die wichtigsten Lösungen für mehr Agilität in der Verwaltung genannt. Das Fazit der Praxis-Beobachtungen lässt sich kurz zusammenfassen: der Digitalisierung wird bisher als Modernisierungsaufgabe der öffentlichen Verwaltung mehr Priorität eingeräumt als der organisationsund fachübergreifenden Umsetzung von Agilitätskonzepten (BearingPoint 2015).

Dabei ist unstreitig, dass Agilität als Führungs- und Managementansatz weit über die Anwendung agiler Methoden für einzelne Projekte hinausgeht. Neben der Literaturrecherche bestätigt dies auch ein Blick auf die herrschende Managementpraxis. Kategorisiert man beispielsweise die entsprechenden Handlungshinweise der drei umsatzstärksten Unternehmensberatungen, McKinsey, Boston Consulting Group und Bain \& Company (Wouter et al. 2018; Pardasani et al. 2018; Rigby et al. 2018), so lassen sich auf drei Ebenen Agilitätsmerkmale definieren, auf die sich eine agile Transformation richtet (siehe Tab. 1). 
Tab. 1 Typische Handlungsfelder für die agile Transformation von Organisationen

\begin{tabular}{|c|c|c|}
\hline Handlungsebene & Merkmale & Agile Managementpraxis \\
\hline Strukturen & $\begin{array}{l}\text { Regeln und Ressourcen können } \\
\text { regelmäßig schnell verändert } \\
\text { werden }\end{array}$ & $\begin{array}{l}\text { - Flache Aufbauorganisation } \\
\text { - Modulare Organisation } \\
\text { - Selbststeuernde cross- } \\
\text { funktionale Teams } \\
\text { - Iterative Lernprozesse } \\
\text { - Transparenz sämtlicher rele- } \\
\text { vanter Information }\end{array}$ \\
\hline Management/Führung & $\begin{array}{l}\text { Entscheidungen werden flexibel } \\
\text { geändert, um Opportunitäten } \\
\text { im Sinne des gemeinsamen } \\
\text { Purpose zu nutzen }\end{array}$ & $\begin{array}{l}\text { - Strategiefindung als perma- } \\
\text { nenter Prozess } \\
\text { - Klar kommunizierter Purpose } \\
\text { - Leadership } \\
\text { - Flexible personelle } \\
\text { Ressourcen } \\
\text { - Variables Rollenverständnis } \\
\text { - Unternehmerisches Denken } \\
\text { in der gesamten Organisation }\end{array}$ \\
\hline Technik & $\begin{array}{l}\text { Software, die Mitarbeitende } \\
\text { befähigt und datenbasierte } \\
\text { Mehrwert-Services für die } \\
\text { Bürger ermöglicht }\end{array}$ & $\begin{array}{l}\text { - Anpassungsfähige modulare } \\
\text { IT-Architektur } \\
\text { - IT-Tools für umfassende } \\
\text { Kollaboration mit internen } \\
\text { und externen Beteiligten } \\
\text { - Kontinuierliches Testen und } \\
\text { Entwickeln von Produkten } \\
\text { und Prozessen } \\
\text { - IT gestütztes Wissens- } \\
\text { management } \\
\text { - Data Analytics }\end{array}$ \\
\hline
\end{tabular}

Offensichtlich ist, dass - übertrüge man diese Handlungsfelder unverändert auf die öffentliche Verwaltung - schnell Grenzen des angestrebten Wandels sichtbar würden. Insbesondere für die Bereiche der Aufbauorganisation, der schnellen Strategiewechsel oder der Personalflexibilität gilt, dass sie in der öffentlichen Verwaltung ganz anderen rechtlichen und politischen Bedingungen unterliegen als in privatwirtschaftlichen Unternehmen. Das betrifft ganz besonders das öffentliche Dienst- und Tarifrecht (BearingPoint 2015, S. 14 f.). Eine analoge Übertragung von Agilitätskonzepten aus dem allgemeinen Management in die öffentliche Verwaltung ist deshalb nicht zielführend. Genauso wenig ist es aber sinnvoll, eine Übertragbarkeit gänzlich zu verneinen. 
Für die Entwicklung einer agilen Verwaltung muss deshalb ein anderer theoretischer bzw. konzeptioneller Ausgangspunkt genommen werden, der weniger den üblichen Engineering-Konzepten mit ihren ganzheitlichen, simultan umzusetzenden Handlungsfeldern folgt, sondern vielmehr davon ausgeht, dass agile Verwaltung zuallererst ein neues Führungshandeln braucht, um dann aus der klassischen top-down-Perspektive Veränderungen in Bezug auf Strukturen und Technik vorzunehmen. Oder anders gesagt: Ohne neue (kommunikative) Führung keine agile Verwaltung. Vor diesem Hintergrund wird der Ansatz einer kommunikationszentrierten Unternehmensführung (Rüegg-Stürm und Grand 2017; Buchholz und Knorre 2019) im Folgenden unter Bezugnahme auf die öffentliche Verwaltung kurz skizziert.

Verfolgt man Agilität als Handlungskonzept der Digitalisierung in Organisationen der öffentlichen Verwaltung, dann geht es neben den reinen Outputzielen aus der Bürgerperspektive, also im Wesentlichen einer besseren Servicequalität, zugleich um die vorgelagerten strategischen Ziele aus der Ressourcenperspektive. Hier steht die Frage im Mittelpunkt, wie sich die internen Ressourcen der öffentlichen Verwaltung noch besser ausschöpfen lassen, um die Outputziele zu erreichen. $\mathrm{Zu}$ diesen grundsätzlich nie ausgeschöpften internen Ressourcen zählen - wie in Unternehmen - an erster Stelle die Mitarbeitenden mit ihrem spezifischen Wissen, Kompetenzen und Verbindungen, auf deren Mobilisierung die interne Kommunikation zielt. Sämtliche Qualitäts- bzw. Exzellenzkriterien agiler Organisationen beruhen darauf, dass die internen Ressourcen möglichst umfassend gehoben werden.

Dabei bezieht sich diese Mobilisierung der internen Ressourcen nicht mehr nur auf die klassischen Ziele des Wissens- und Qualitätsmanagements, also implizites Wissen in explizites umzuwandeln oder Nullfehler-Qualitäten zu erzielen. Gemäß dem hier nachvollzogenen kommunikationszentrierten Managementkonzept kommt es vielmehr darauf an, dass Organisationen die sie umgebende Umwelt bzw. die dort wirkenden Stakeholder in ihre Wertschöpfungsprozesse grundsätzlich miteinbeziehen. Führung hat den Anspruch durch reflektierendes Führungshandeln diesen Abgleich zwischen Organisationen, hier Behörden, und ihrer Umwelt so zu gestalten, dass die Zwecke und Ziele der Organisation trotz einer oder gerade in einer VUCA-Umwelt erreicht werden können. Treibt man diesen Gedanken auf die Spitze, dann können agile Organisationen die schwierigen, komplexen Umweltbeziehungen zu einer quasi-internen Ressource machen, die sie mithilfe ihrer Mitarbeitenden für ihre Zwecke und Ziele aktivieren.

Dies erfordert eine grundsätzliche Wachsamkeit gegenüber den Veränderungen in einer volatilen Umwelt ebenso wie im Hinblick auf veränderte Bewertungen der internen Ressourcen und Abläufe. Als fundamentale Bedingung agilen 
Handelns gilt seit einigen Jahren bereits ein systemisches und systematisches Beobachten und Zuhören, und zwar in der Organisation genauso wie bei Kunden und Partnern (Knorre 2012, S. 17 ff.). Wachsamkeit ist aber nur dann umsetzungsfähig, wenn sie auf den entsprechenden Kompetenzen und Freiräumen der Organisationsmitglieder fußen kann, auf Mitarbeitende und Führungskräfte gleichermaßen. Diese organisationale Wachsamkeit ist zugegebenermaßen in Behörden besonders schwierig, denn alle Beobachtungen der externen Umwelt, die irritieren können, treffen dort auf einen sehr starken Referenzrahmen (Rüegg-Stürm und Grand 2017, S. 178 ff.; Buchholz und Knorre 2019, S. 18 f.) bestehender Regeln und Deutungen, die eine Fülle von ,blinden Flecken“ verursachen.

Dennoch bleibt das Postulat, dass auch die öffentliche Verwaltung sich grundlegend um mehr Wachsamkeit bemühen muss, will man agiler werden. Auch für die öffentliche Verwaltung gilt, dass Führung sich vor dem Hintergrund der Anforderungen der Agilität ändern muss. Führung in der öffentlichen Verwaltung ist analog zu privatwirtschaftlichen Organisationen grundsätzlich prozessual anzulegen, auf Stakeholder bezogen und durch kommunikative Auseinandersetzungen bzw. Diskurse zu gestalten. Agile Führung verbindet die Ebenen der Umwelt, der Organisation und des Managements zu einem effektiven Verwaltungshandeln, das mittels Kommunikation gestaltet wird. „Neben den gelernten Managementdreisatz aus analysieren, planen und umsetzen treten drei neue kommunikationszentrierte Handlungen der Unternehmensführung: zuhören, sinnstiften, reflektieren" (Buchholz und Knorre 2019, S. 21). Diese Schlussfolgerung gilt genauso für die Organisationen der öffentlichen Verwaltung.

Agilität erweist sich deshalb vor allem als kommunikationszentriertes Handeln, das auf den Führungsetagen beginnt. Dieser Ausgangspunkt für die agile Transformation von Organisationen verspricht auch für die öffentliche Verwaltung Aussicht auf Erfolg, ganz einfach, weil er es vermeidet, an den Punkten zuerst anzusetzen, an denen die Hindernisse besonders hoch sind - etwa durch das Dienstrecht, das einem flexiblen Ressourceneinsatz oder einem multiplen Rollenverständnis entgegensteht. Für die agile Transformation, die auf Führungshandeln beruht, hat sich die Ausgestaltung von vier Steuergrößen herausgestellt. Diese sind definiert als Purpose (Sinn und Orientierung), Mindset (Einstellung und Handlungslogik), Relation (Kollaboration und Vernetzung) und Kompetenz (Wissen und Vielfalt) (Buchholz und Knorre 2019, S. 26 ff.). Diese Steuergrößen müssen durch Führung verändert werden, um agiles organisationales Handeln zu erzielen.

Die Steuergröße Purpose fokussiert wertebasiertes, sinnvolles organisationales Handeln und vermittelt das bei aller Veränderung Beständige. Mindset verweist auf die entsprechende, auf Purpose ausgerichtete agile Denk- und Handlungslogik und vermittelt dazu die grundsätzliche Veränderbarkeit von 
Gegebenheiten im organisationalen Handeln (Kontingenz). Die Steuergröße Relation lenkt die Aufmerksamkeit auf die agile Zusammenarbeit mit internen und externen Stakeholdern, die in Form von Kollaboration und Vernetzung gelebt wird. Diese ermöglicht eine wachsame Beobachtung aller internen und externen Veränderungen und erlaubt es, daraus Schlussfolgerungen für die Entwicklung der Organisation abzuleiten. Schließlich sind besondere Kompetenzen der Organisationsmitglieder sowie ein kontinuierlicher Wissenstransfer innerhalb der Organisation zu entwickeln. Das betrifft nicht zuletzt das Agilitätswissen (Buchholz und Knorre 2012, S. 14 ff.) selbst.

\section{Agilität in der öffentlichen Verwaltung: Merkmale kommunikationszentrierter Führung und deren Relevanz für die interne Kommunikation}

Diese Steuergrößen sind im Kontext von öffentlicher Verwaltung mit etwas anderen Stellgrößen zu beeinflussen als in privatwirtschaftlichen Unternehmen. Jedoch lassen sich analog zum agilen Management ebenfalls vier aufeinander bezogene Handlungsfelder identifizieren, in denen die interne Kommunikation jeweils eine der zentralen Stellgrößen bildet.

\section{Handlungsfeld Purpose}

Es versteht sich von selbst, dass Purpose gerade im öffentlichen Sektor im Grunde als gegebene Größe zu sehen ist. Die Gemeinwohlorientierung der öffentlichen Verwaltung, die den Willen des parlamentarischen Gesetzgebers umsetzt, ist so gesehen als ein Vorteil auf dem Weg zu mehr Agilität anzusehen. Allerdings sind die Zwecke, denen die jeweilige Behörde explizit dient, oft nicht hinreichend kommuniziert und zu wenig in den Kontext Sinn stiftender Narrative gestellt - vielleicht, weil es für selbstverständlich gehalten wird, was Sinn und Zweck des eigenen Tuns ist.

Die Führungsaufgabe besteht nun darin, dafür zu sorgen, dass die Organisation die guten Zwecke, denen sie dient, nicht kollektiv aus den Augen verliert, sondern dass diese im Sinne einer Selbstvergewisserung kontinuierlich intern kommuniziert werden. Wer Mitarbeitende mobilisieren will, damit sie als Botschafter einer öffentlichen Organisation auftreten und Vertrauen zurückgewinnen, der darf keinen Moment Unsicherheit über Sinn und Zweck der eigenen Rolle und des eigenen Handelns aufkommen lassen. Das gilt umso mehr, wenn gewünscht ist, dass Mitarbeitende mehr Eigeninitiative zeigen, dass sie neue Ideen einbringen und Beobachtungen aus ihrem jeweils spezifischen Stakeholderumfeld weitergeben. 


\section{Handlungsfeld Organisationskultur}

Vergleichsweise komplexer gestaltet sich im Kontext von öffentlicher Verwaltung die Steuergröße Mindset. Es geht weniger um einfaches, agiles Methodenwissen, als vielmehr um das Durchdringen der Handlungslogik der Agilität. Diese leitet sich grundlegend aus dem Denken in Kontingenzen ab, also der Annahme, dass es zu jeder Entscheidung mindestens eine ebenfalls sinnvolle Alternative gibt, die die einmal getroffene Entscheidung herausfordert. Insbesondere durch den unvermeidlichen Abschied von der Kontrollillusion zugunsten eines pragmatischen, iterativen und inkrementellen Vorgehens mit hoher Fehlertoleranz entstehen duale, oft paradoxe Anforderungen an agiles Verwaltungshandeln, die durchaus im Gegensatz zum Effizienzprinzip des Public Management stehen.

Agile Führung sorgt dementsprechend dafür, dass neben den Pflichtaufgaben der öffentlichen Verwaltung immer auch Küraufgaben (Prodoehl 2014, S. 233 ff.), neben geplanten Maßnahmen immer auch ungeplante Opportunitäten, neben stabilen Regeln immer auch flexible ad-hoc Entscheidungen ihren Platz haben. Diese Beidhändigkeit oder Ambidextrie ist das prägende Merkmal agiler Organisationen und damit Gestaltungsauftrag der kommunikationszentrierten Führung. Er erstreckt sich im Übrigen über die hier fokussierte Organisationskultur hinaus auch auf die Organisationsgestaltung, indem hierarchische und heterarchische Strukturelemente miteinander verknüpft werden (Buchholz und Knorre 2019, S. 75 ff. sowie S. 249 f.). Strenge Hierarchien und flexible, oft informelle interne Netzwerke bestehen gleichzeitig und erfüllen jeweils unterschiedliche Funktionen für die agile Organisation. Die einen sorgen für Stabilität und eindeutige Verantwortung, die anderen für schnelle, organisations- und ressortübergreifende Abstimmungen und Innovationen.

All dies ist nicht nur äußerst erklärungsbedürftig für die Mitarbeitenden, sondern verlangt darüber hinaus kulturbildende Artefakte und Werte bzw. Regeln, die mit Unterstützung der internen Kommunikation gestaltet werden. Für die kommunikationszentrierte Führung sind geeignete Narrationen und Instrumente zu finden, mit denen sowohl Mitarbeitenden als auch Führungskräften selbst bei mehrdeutigen und unsicheren Situationen jederzeit eine Ausrichtung am Purpose der Organisation sowie den spezifischen Zielen, zum Beispiel eines Regierungsprogramms, möglich ist.

\section{Handlungsfeld Kollaboration und Vernetzung}

Die Stellgrößen Kollaboration und Vernetzung verweisen in der internen Perspektive auf ein auch in der öffentlichen Verwaltung bekanntes Aufgabenfeld, nämlich den Versuch, die Nachteile hierarchischer Systeme durch den Einbau heterarchischer Elemente, vor allem in Form von Projekten und abteilungsübergreifenden 
Teams, auszugleichen. Gerade die organisations- und behördenübergreifende Zusammenarbeit ist nicht zuletzt in der Selbsteinschätzung nach wie vor ein Schwachpunkt in der öffentlichen Verwaltung (BearingPoint 2015, S. 14). Projekte sind nicht nur ,durchzuziehen', sondern es gilt, für sie mittels Kommunikation interne Unterstützung zu mobilisieren.

In der vorrangig externen Perspektive geht es im Handlungsfeld Relation um die o. g. Wachsamkeit für Veränderungen in der von Stakeholdern gestalteten Umwelt, die Organisationen der öffentlichen Verwaltung bzw. deren Mitarbeitenden zu leisten imstande sein müssen. Outside-In-Kommunikation (Buchholz und Knorre 2019, S. 221 f.) gehört deshalb zu den zentralen Aufgaben in diesem Handlungsfeld, die in vielen Gestaltungsvarianten daherkommen kann, vom regelmäßigen Personalaustausch über Stakeholderdialoge bis hin zu Sound Boards oder Kundenbeiräten. Wachsamkeit lässt sich als organisationale Kompetenz umso besser entwickeln als sie im Sinne des kollektiven Lernens möglichst viele Organisationsmitglieder unabhängig von Hierarchiestufen miteinbezieht. Dies erfordert neben der Outside-In-Kommunikation immer zugleich eine interne Kommunikation, die entsprechend mobilisieren kann.

\section{Handlungsfeld Kompetenzen}

Als ressourcenorientiertes Handlungskonzept verweist Agilität darauf, welche zentrale Bedeutung dem Wissen der Mitarbeitenden und der Motivation, dieses Wissen auch zu nutzen, zukommt. Agilität ist geprägt durch Lernen, Wissen zu teilen und dieses immer wieder zu hinterfragen.

Agile Organisationen sind grundsätzlich als lernende Organisationen zu verstehen, wobei agiles Lernen nicht davon ausgeht, dass eine Situation im Vorfeld vollständig analysiert und verstanden werden kann. Das notwendige Wissen für den nächsten Schritt generiert sich durch ergebnisoffenes Handeln der Akteure, eben durch Reflexion, Experimentieren und Testen. Agiles Lernen findet keinesfalls nur in Expertenzirkeln statt, sondern es gilt der Grundsatz: je vielfältiger die Beteiligten sind, desto besser für das gemeinsame bzw. organisationale Lernen. Gerade die Verbindung zwischen Expertenwissen und vielen anderen Perspektiven, die Mitarbeitende einbringen können, zum Beispiel durch ihre Praxiserfahrungen im Kunden- bzw. Bürgerkontakt, macht das Spezifische des agilen Lernens aus.

Insgesamt verlangen Veränderungen im Sinne des Agilitätskonzeptes einen hohen Grad an kritischer Selbstreflexion, angefangen bei den Führungskräften. Wenn die gefundenen Lösungen grundsätzlich immer auch anders aussehen könnten, dann ist Management nicht mehr vorrangig als plangesteuerter Gestaltungsanspruch definiert. Vielmehr geht es geradezu umgekehrt darum, 
Entscheidungen und die daraus entstandenen Regeln und Ressourcenverteilungen systematisch infrage zu stellen. „Hierzu notwendig ist Reflexion, welche die eingespielte organisationale Wertschöpfung in ihrem alltäglichen Vollzug unterbricht, aus Distanz kollektiv hinterfragt und kommunikativ bearbeitet" (RüeggStürm und Grand 2017, S. 196 f.).

Das führt direkt zur zweiten Aufgabe in diesem Handlungsfeld. Diese besteht salopp gesagt darin, mit hoher Reichweite in der Organisation zu erklären, wie Agilität funktioniert. Es handelt sich also um Meta-Lernen, d. h. die Fähigkeit, Lernen zu lernen. Diese Art von Agilitätswissen, d. h. das Wissen, Verstehen und Akzeptieren von Kontingenzen und Emergenzen (mithin die Abkehr vom monokausalen Denken) ist nicht zuletzt durch entsprechende interne Kommunikation aufzubauen.

Bei der Umsetzung der kommunikationszentrierten Führung in den genannten Handlungsfeldern kann helfen, dass die öffentliche Verwaltung mit der politischen Spitze zwischen den politisch besetzten Steuerungseinheiten einerseits und dem (verbeamteten) Verwaltungsapparat andererseits bereits über ein duales Steuerungssystem verfügt. Denn nach wie vor gilt das Errichten eines ,zweiten Betriebssystems' (Kotter 2014), dass das hierarchische Entscheidungssystem um agile Elemente ergänzt, als Erfolgsrezept für die gesteuerte Veränderung von Organisationen. Die agile Transformation lässt es also sinnvoll erscheinen, die Dualität aus politischer Spitze und Verwaltung eher als Chance denn als lästigen Effizienzverlust (Bogumil 2010) zu bewerten. Die Zukunftsaufgabe wird darin bestehen, aus dieser Dichotomie eine funktionierende Ambidextrie zu machen, die agiles Handeln der öffentlichen Verwaltung beschleunigen kann.

Das Zusammenwirken von zwei Steuerungssystemen in einer Organisation ist darüber hinaus grundsätzlich ein wichtiger Gedanke aus dem Agilitätskonzept, der für die öffentliche Verwaltung nutzbringend einzusetzen ist. Wie es aus dem Innovationsmanagement bekannt ist, lassen sich auch in der öffentlichen Verwaltung kleinere, schnellere Organisationseinheiten schaffen, die das notwendige regelgebundene, hierarchische Steuerungssystem ergänzen und damit die Organisation insgesamt agiler machen. Dann allerdings kommt der internen Kommunikation eine weitere Aufgabe zu, nämlich die kommunikativen Verbindungen zwischen den beiden Systemen herzustellen, sodass das organisationale Handeln insgesamt profitieren kann (Buchholz und Knorre 2017, S. 17 f., 2019, S. 101 ff.).

Wann immer in den vorangegangenen Überlegungen zu Agilität von interner Kommunikation die Rede war, dann geht es nur in zweiter Linie um die in der öffentlichen Verwaltung ebenfalls ausgeprägte Fokussierung auf den (digitalen) Medieneinsatz bzw. die operativen Kommunikationsmaßnahmen, zum Beispiel Social Intranet, Messengerdienste, Mitarbeiterapps oder Blogs. Interne 
Kommunikation ist vielmehr eine Führungsfunktion, die ,zuständigkeitshalber ‘ von Kommunikationsexperten übernommen wird, deren professionelle Beiträge zur Agilität in den genannten Handlungsfeldern klar definiert sind. Die Stichworte hier lauten Unterstützung von organisationaler Wachsamkeit, Vernetzung, Orientierungssicherheit und Leadership (Buchholz und Knorre 2017, S. 27 ff.).

Interne Kommunikation ist aber jenseits der zuständigen Organisationseinheit zugleich Teil der hier beschriebenen kommunikationszentrierten Führung in agilen Organisationen insgesamt.

\begin{abstract}
„Warum berichte ich beim Thema ,Zielorientierte Steuerung ${ }^{\natural}$ über die Instrumente der internen Kommunikation? Weil sie meiner Erfahrung nach entscheidend dafür sind, dass die Strategie mehr wert ist als das Papier, auf dem sie geschrieben steht. Nur wenn wir in unsere Institutionen hineinwirken, wenn wir unsere Beschäftigten mitnehmen auf die strategische Reise, nur dann haben wir eine Chance, das Reiseziel zu erreichen.“(Weidmann 2014)
\end{abstract}

Agilität ist nun aber mehr als nur das hier zitierte „,[M]itnehmen“ (Weidmann 2014) von Mitarbeitenden. Es ist auch mehr als nur das Weiterkommunizieren von Entscheidungen aus der Führungsetage. Eine kommunikationszentrierte Führung, die agile Organisationen kennzeichnet, bedeutet eine durchgehende Betrachtung und Beachtung kommunikativer Beziehungen als Gestaltungsprinzip in und von Organisationen. Denn mit diesem kommunikationszentrierten Konzept lassen sich die internen Ressourcen heben und für agiles Behördenhandeln mobilisieren. Das ist das Handlungsfeld der internen Kommunikation.

\title{
$4 \quad$ Fazit
}

Zweifelsohne sind die Zielkonflikte bzw. Paradoxien, mit denen Organisationen der öffentlichen Verwaltung umgehen müssen, noch deutlich ausgeprägter als in privatwirtschaftlichen Unternehmen. Das gleichzeitige Streben nach Beständigkeit, Nachvollziehbarkeit oder gerichtsfesten Prozessen einerseits und einem schnellen Reagieren auf neue Themen oder gar Krisen von unabsehbarer Art und unbekanntem Ausmaß andererseits ist hier besonders schwierig. Ohne Prophet sein zu müssen, lässt sich vorhersagen, dass diese Zielkonflikte eher mehr als weniger werden, wie zum Beispiel die in diesem Band beschriebenen Formen frühzeitiger Bürgerbeteiligung (siehe den Beitrag von Rademacher und Kollegen in diesem Band) zeigen, dass die Entscheidungsprozesse über Infrastrukturprojekte zunächst einmal 
weiter zeitlich gestreckt werden, obwohl sie eigentlich schnell und pragmatisch realisiert werden müssten.

Umso wichtiger ist es, dass auch in der öffentlichen Verwaltung Agilität als Handlungskonzept aus der unterkomplexen Methodenperspektive befreit und stattdessen vor allem als basales, ressourcenorientiertes und kommunikationszentriertes Führungskonzept interpretiert wird. Das gilt zunächst für die politisch-strategische Führung auf der Ebene der Ministerien sowie obersten Behörden, die sich als agile Administration aufstellen können, indem sie systematisch die vier genannten Steuergrößen durch die genannten Handlungsoptionen verändern. Doch selbst in rechtlich fixierten Verwaltungsabläufen nachgeordneter Umsetzungsorganisationen kann die Agilität von Verwaltungshandeln erhöht werden, indem das Agilitätskonzept sowohl in seiner strategischen als auch methodischen Dimension angewendet wird. Blickt man noch einmal auf die ehrgeizigen Digitalisierungspläne der öffentlichen Verwaltung, dann werden diese Postulate immer dringender.

Natürlich bilden insbesondere das Dienst- und Tarifrecht erhebliche Hindernisse auf dem Weg zu einer agilen Verwaltung, selbstverständlich ist eine ressortübergreifende Zusammenarbeit besonders in Koalitionsregierungen schnell an ihre Grenzen gelangt und offensichtlich verschlingen große Projekte nach wie vor viel zu viel Ressourcen. Aber wenn es stimmt, dass eine Vertrauenskrise zwischen staatlichen Institutionen bzw. deren politischer Führungsspitze und den Bürgern besteht, dann ist die kommunikationsbasierte Mobilisierung von Mitarbeitenden der öffentlichen Verwaltung im Sinne des Agilitätskonzeptes einer der wenigen Erfolg versprechenden Handlungsansätze, diese Vertrauenskrise zu überwinden. Eine agile Verwaltung kann dazu beitragen, einer ,,Verselbstständigung der Exekutive“ (Winkler 2018, S. 2) und der daraus resultierenden „Entfremdung der Regierenden von der Gesellschaft“ (Winkler 2018, S. 2) entgegenzuwirken.

\section{Literatur}

Bartonitz, M., Lévesque, V., Michl, T., Steinbrecher, W., Vonhof, C., \& Wagner, L. (Hrsg.). (2018). Agile Verwaltung. Wie der Öffentliche Dienst aus der Gegenwart die Zukunft entwickeln kann. Wiesbaden: Springer Gabler.

BearingPoint. (2015). Business Agility. Bedeutung von Agilität in der Verwaltung. https:// www.bearingpoint.com/files/BEDE13_0860_WP_DE_AgileVerwaltung_final_web. pdf?hash=e1355fd054b47771f8970883f46226100db8b7496bdf66d5. Zugegriffen: 14. Jan. 2019.

Bogumil, J. (2010). Die politische Führung öffentlicher Dienste: Möglichkeiten und Grenzen der Reorganisation. In R. Koch, P. Conrad, \& H. W. Lorig (Hrsg.), New public service (S. 111-1282). Wiesbaden: Gabler. 
Buchholz, U., \& Knorre, S. (2012). Interne Unternehmenskommunikation in resilienten Organisationen. Berlin: Springer Gabler.

Buchholz, U., \& Knorre, S. (2017). Interne Kommunikation in agilen Unternehmen. Eine Einführung. Wiesbaden: Springer Gabler.

Buchholz, U., \& Knorre, S. (2019). Interne Kommunikation und Unternehmensführung. Theorie und Praxis eines kommunikationszentrierten Managements. Wiesbaden: Springer Gabler.

Bundesverwaltungsamt. (2015). OfficeNet $\left(\mathrm{ON}^{2}\right)$. https://www.bva.bund.de/DE/Services/Behoerden/Beratung/Beratungszentrum/Methoden/_documents/stda_officenet_ vm.html. Zugegriffen: 14. Jan. 2019.

Dörfel, L., Mann, P., Boesler, B., Rosenzweig, U., \& Herbst, G. (2016). Trendmonitor interne Kommunikation. Entwicklung und Potenziale des Berufsstandes. https://content-marketing-forum.com/wp-content/uploads/2017/01/MPM_Trendmonitor_A5_ Langfassung_Ansicht.pdf. Zugegriffen: 12. Dez. 2018.

Döring-Seipel, E., \& Lantermann, E.-D. (2015). Komplexitätsmanagement. Psychologische Erkenntnisse zu einer zentralen Führungsaufgabe. Wiesbaden: Springer Gabler.

Edelman. (2017). Trust Barometer 2017. https://www.edelman.de/newsroom/studien-insights/das-fundament-der-unternehmenskommunikation/. Zugegriffen: 12. Dez. 2018.

EFQM. (2013). EFQM excellence model. http://www.efqm.org/index.php/efqm-model-2013/. Zugegriffen: 14. Jan. 2019.

Goldstein, B., \& Dyson, L. (Hrsg.). (2013). Open data and the future of civic innovation. http://beyondtransparency.org/pdf/BeyondTransparency.pdf. Zugegriffen: 1. Okt. 2018.

Hill, H. (2014). Zukunftsfähige Verwaltungsentwicklung. Ein Aufruf zur Gestaltung. Jahrbuch der Schweizerischen Verwaltungswissenschaften, 5, 11-26.

Hill, H. (2017). Die zukunftsfähige Verwaltung. Ein Nachfragetool für CAF. https://www. uni-speyer.de/files/de/Lehrst\%C3\%BChle/Hill/Publikationen/Diezukunftsf\%C3\%A4higeVerwaltung.pdf. Zugegriffen: 12. Dez. 2018.

Huck-Sandhu, S. (2016). Interne Kommunikation im Wandel: Entwicklungslinien, Status Quo und Ansatzpunkte für die Forschung. In S. Huck-Sandhu (Hrsg.), Interne Kommunikation im Wandel. Theoretische Konzepte und empirische Befunde (S. 1-19). Wiesbaden: Springer VS.

Knorre, S. (2012). Interne Unternehmenskommunikation aus der Perspektive organisationaler Resilienz. In G. Bentele, M. Piwinger, \& G. Schönborn (Hrsg.), Kommunikationsmanagement. Strategien, Wissen, Lösungen. Neuwied: Luchterhand (Loseblattsammlung Lieferung 3.90).

Koch, R. (2010). Theorieproduktionen und Gestaltungsoptionen in der Modernisierung öffentlicher Dienste. In R. Koch, P. Conrad, \& H. W. Lorig (Hrsg.), New public service (S. 13-39). Wiesbaden: Gabler.

Kortendick, O., \& Dyrks, T. (2018). V-Modell XT „,agil“. Erfahrungen mit der Einführung von agilen Elementen in der Qualitätssicherung. https://fg-tav.gi.de/fileadmin/FG/ TAV/41.TAV/41_GI-TAV_paper_5.pdf. Zugegriffen: 12. Dez. 2018.

Kotter, J. P. (2014). Accelerate. Building strategic agility for a faster-moving world. Boston: Harvard Business Review Press.

Levesque, V., \& Steinbrecher, W. (2017). Agile Arbeitsmethoden in der öffentlichen Verwaltung. Innovative Verwaltung, 5, 28-30. 
Mack, O., Khare, A., Kramer, A., \& Burgartz, T. (Hrsg.). (2016). Managing in a VUCA world. Heidelberg: Springer.

Pardasani, N., Danoesastro, M., Alfrink, K., Stutts, L., Schotkamp, T., \& Hilbers, P. (2018). Taking agile transformations beyond the tipping point. https://www.bcg.com/de-de/ publications/2018/taking-agile-transformations-beyond-tipping-point.aspx. Zugegriffen: 14. Jan. 2019.

Prodoehl, H. G. (2014). Synaptisches Management. Strategische Unternehmensführung im 21. Jahrhundert. Wiesbaden: Springer Gabler.

Rigby, D. K., Sutherland, J., \& Noble, A. (2018). Agile at scale. Harvard Business Review May-June, 88-96.

Rüegg-Stürm, J., \& Grand, S. (2017). Das St. Galler Management-Modell (3. Aufl.). Bern: Haupt.

Weidmann, J. (2014). Auch ohne Glaskugel strategisch planen. Zur Bedeutung einer zielorientierten Steuerung in der deutschen Verwaltung am Beispiel der Bundesbank. https://www.verwaltung-innovativ.de/SharedDocs/Reden/DE/2014/rede_Weidmann. html. Zugegriffen: 18. Dez. 2018.

Winkler, H. A. (2018). Deutschlands Öffnung gegenüber dem Westen ist nicht abgeschlossen. Welt am Sonntag, 30, 2.

Wouter, A., De Smet, A., Lackey, G., Lurie, M., \& Murarka, M. (2018). The five trademarks of agile organizations. https://www.mckinsey.de/publikationen/leading-in-a-disruptive-world/the-five-trademarks-of-agile-organizations. Zugegriffen: 14. Jan. 2019.

Zerfass, A., Tench, R., Verhoeven, P., Verčič, D., \& Moreno, A. (2018). European communication monitor 2018. http://www.communicationmonitor.eu/2018/06/13/ecm-european-communication-monitor-2018. Zugegriffen: 12. Dez. 2018.

Open Access Dieses Kapitel wird unter der Creative Commons Namensnennung 4.0 International Lizenz (http://creativecommons.org/licenses/by/4.0/deed.de) veröffentlicht, welche die Nutzung, Vervielfältigung, Bearbeitung, Verbreitung und Wiedergabe in jeglichem Medium und Format erlaubt, sofern Sie den/die ursprünglichen Autor(en) und die Quelle ordnungsgemäß nennen, einen Link zur Creative Commons Lizenz beifügen und angeben, ob Änderungen vorgenommen wurden.

Die in diesem Kapitel enthaltenen Bilder und sonstiges Drittmaterial unterliegen ebenfalls der genannten Creative Commons Lizenz, sofern sich aus der Abbildungslegende nichts anderes ergibt. Sofern das betreffende Material nicht unter der genannten Creative Commons Lizenz steht und die betreffende Handlung nicht nach gesetzlichen Vorschriften erlaubt ist, ist für die oben aufgeführten Weiterverwendungen des Materials die Einwilligung des jeweiligen Rechteinhabers einzuholen.

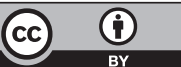

